\title{
Determination of Potassium Levels in Bananas Using an Optical Sensor with a Flat and Concave Mirror Plane
}

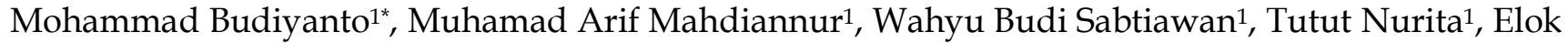 \\ Sudibyo ${ }^{1}$
}

${ }^{1}$ Undergraduate Program in Science Education, Department of Natural Science, Faculty of Mathematics and Natural Sciences, Universitas Negeri Surabaya, Surabaya, East Java, Indonesia.

DOI: $10.29303 /$ jppipa.v7i3.703

\section{Article Info}

Received: March 29th, 2021

Revised: May 18th, 2021

Accepted: May 22th, 2021

\begin{abstract}
Potassium is a nutrient that plays a role in maintaining the function of the muscles and nerves that control the heart and is needed for body stability. The potassium content in the body can be obtained from foods such as bananas. The development of instruments and methods that are developed to obtain a more accurate measurement of potassium concentration requires an instrument that has high linearity and sensitivity. The instrument is in the form of an optical sensor system equipped with the use of optical fibers to guide the waveform to maintain its intensity stability. In this study, an experimental method was conducted with a sample of a standard solution with potassium as the solute and pure water as the solvent. Then continue to measurements on samples of banana milk and green banana fruit extracts. The results of the analysis of the measurement data using an optical sensor with a concave mirror reflection plane obtained a sensitivity of 0.36 $\mathrm{mV} / \mathrm{ppm}$ and a linearity of $82.56 \%$. In the plane of the flat mirror reflection, obtained an optical sensor with a plane mirror reflection plane shows a sensitivity of $0.12 \mathrm{mV} / \mathrm{ppm}$ and a linearity of $97.6 \%$. The highest and most accurate linearity value is found in the plane mirror plane results. The next stage is the result of the maximum output voltage read on the optical detector through an optical sensor with a sample of extracts of milk banana and green banana. The results of data analysis on the linear equation with the highest linearity obtained the potassium content in milk bananas of $391.54 \mathrm{ppm}$ and the green banana extract solution obtained $307.91 \mathrm{ppm}$, so it can be concluded that the potassium content in milk bananas is higher than green bananas with a linearity of more than $97 \%$.
\end{abstract}

Keywords: Potassium; optical sensor; flat mirror; concave mirror; banana.

Citation: Budiyanto, M., Mahdiannur, M., Sabtiawan, W., Nurita, T., \& Sudibyo, E. (2021). Determination of Potassium Levels in Bananas Using an Optical Sensor with a Flat and Concave Mirror Plane. Jurnal Penelitian Pendidikan IPA, 7(3). 293-297. doi:https://doi.org/10.29303/ippipa.v7i3.703

\section{Introduction}

Potassium is one of the nutrients the body needs and has a significant role in keeping fluid balance in the human body. The recommended need for potassium consumption for adults is between 4500 - $4700 \mathrm{mg}$ per day. The presence of potassium in the body to control blood pressure and maintain muscle and nerve function. Potassium is also known as an essential mineral as an electrolyte. The fulfillment of potassium in the body is obtained by consuming foods in the form of fruits such as bananas.

The potassium content in bananas is well known to the public. This is from infancy to adulthood by consuming bananas to meet potassium needs in the hope of supporting the balance of fluids in the body so that it remains in a healthy condition. There are several potential benefits of new fermented raw banana 
powder as a food ingredient for enhancing the body's immunity (Horie et al., 2020). Potassium deficiency can be experienced by a person and can be prevented by eating foods that have high potassium and paying attention to their daily diet.

The existence of a normal body can also be viewed from the condition of potassium in the body, so it is deemed necessary to have an instrument to determine potassium, one of which in this study was carried out using bananas. As a consideration, bananas are easily available in the community and are often consumed with cheap considerations and high potassium content. One of them is green banana by determining the amount of in vitro bioaccessibility of macrominerals and tracking elements in green banana flour (do Prado Ferreira \& Teixeira Tarley, 2020). In this study, two types of bananas will be evaluated for potassium content using an optical sensor, a set of optical sensors that will use variations in the reflected plane, namely flat mirrors and concave mirrors as a comparison which is the more accurate result for determining the potassium content in a banana.

The bananas that are often consumed to fulfill potassium in the body are usually types of milk bananas and green bananas. The potassium content of the two bananas will be examined so that they know how many measurements of potassium levels have been met for the stability and health needs of the human body. Many methods have been used for research to determine potassium levels with various techniques using the rapid colorimetric method (Qiu et al., 2019), an instrument with Raman scattering spectroscopy (Su et al., 2016). This measurement is the principle of the scientific concept is almost the same as using a fraction of a certain substance in a solvent and a solute (Susilawati et al., 2018).

The development of tools and methods that are developed to obtain more valid and exact measurement results for potassium levels must require the instrument to have a measurement output with high sensitivity. The development of this method uses the principle of laser light symptoms through an optical fiber-assisted optical sensor system. Optical fiber is an electromagnetic wave transmission channel that uses exceptionally fine glass and plastic materials to transmit light waves. Light sources often use laser light such as the Helium Neon Laser. Light in optical fibers does not come out because the refractive index of glass is greater than the refractive index of air, because the laser has a very narrow spectrum like a fiber coupler (Samian et al., 2018).

The application of optical sensors has been developed by several researchers to figure out the content of certain substances in food either in powder or solution form. As in fiber optic sensors based on wave intensity with the aim of determining calcium (Yasin et al., 2015). Optical sensors with a variety of optical fibers for wave guides to determine the purity of honey (Hida et al., 2013). Development of a multimode sensor design using taper fibers to determine glucose (Yasin et al., 2015). Fiber optic sensor also used to measure salinity (Rahman et al., 2012, 2013). There is also a sensor using two fiber bundles for the levels of a substance in solution (Samian et al., 2018).

Based on the description of the exposure to the potassium content in bananas, the researchers developed an optical sensor to determine potassium levels using a flat and concave mirror plane to display accurate measurement results and better sensor sensitivity.

\section{Method}

Research to find the levels of potassium in bananas was conducted using an experimental method with a preliminary test using standard potassium output with a sample of $0 \mathrm{ppm}$ to $10 \mathrm{ppm}$ and continued with the banana extract sample stage by selecting the equation with the highest linearity. The results of this standard potassium test use a sensor with a flat mirror plane and a concave mirror. The utilization of flat and concave mirror in the detection also used by Rahman et al. (2012) in their research. This two-plane reflective sensor is used to determine the linearity equation results that are more accurate and valid and have high sensitivity. The application of optical sensors to determine standard potassium levels using the principle of fluorescent helium laser light absorption as a source of electromagnetic waves. The fluorescent helium laser beam travels through the optical fiber to reduce the diffuse intensity reduction. The optical fiber guides the propagation of the laser beam to maximize the intensity of the source hitting the sample solution through the receiving optical fiber.

The sample of potassium used in this study was a sample of standard solution with potassium as the solute and pure water as the solvent (sample picture in Figure 2). The sample of the potassium solution in the lower vessel has a flat and concave mirror that reflects the laser light that has been absorbed by the sample solution. The reflected fluorescent helium laser wave is transmitted through the optical fiber of the receiver to be measured by the change in the intensity of the wave using an optical detector. The intensity of the reflected laser light is read by an optical detector and converted to a display of electrical energy by measuring the maximum voltage using a digital voltmeter. The parameters measured in the experiment investigated the relationship between changes in the concentration 
of a sample of potassium solution with the maximum voltage read on a digital voltmeter. The optical sensor performance in research measuring potassium concentration using optical sensors includes sensitivity, linear range, and linearity. The linearity equation of the plane of the flat and concave mirror reflection will be used as a reference for finding the potassium content in the extract solution of milk banana and green banana.

The study was to determine the potassium content in banana extract solution using a He-Ne laser (Rahman et al., 2013) with a wavelength of $632.5 \mathrm{~nm}$, a power of $5 \mathrm{~mW}$. The fluorescent helium laser propagates and anticipates the diffuse intensity reduction which is guided by an optical fiber in order to maximize the intensity of the propagating waves. Standard potassium solution in this study there are six variations of the sample with a concentration of $0,2,4$, 6,8 , and $10 \mathrm{ppm}$. Capture and reception of laser signals reflected by flat and concave mirrors using an optical detector. There is a position micrometer that functions to adjust the shift position changes to get the maximum voltage read on the digital voltmeter. To reduce bias and appropriate tool performance, characterization of the optical sensor is started to determine the shift of the fiber bundle sensing channel towards flat and concave mirrors, which then performs potassium detection. The optical sensor design to determine the potassium content in bananas is shown in Figure 1.

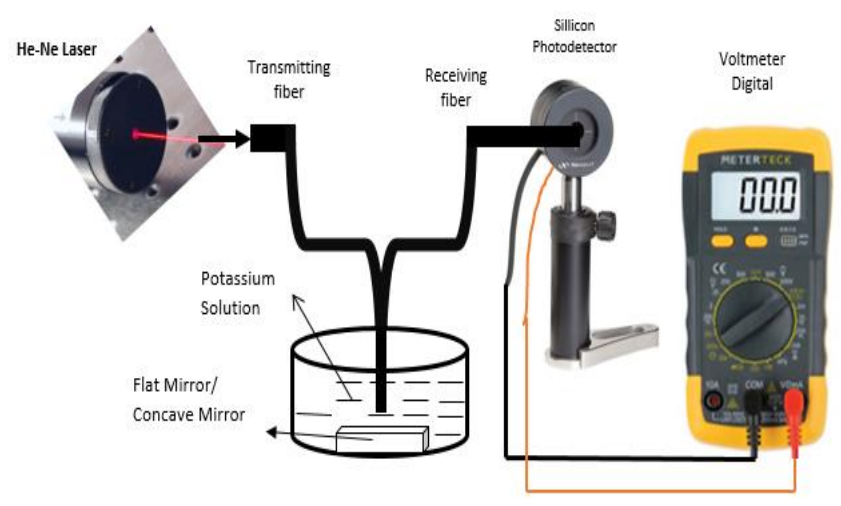

Figure 1. The optical sensor design determines the potassium levels in bananas

Research on potassium levels was conducted by adjusting the shift distance between the optical fiber of the receiving bundle and the sample of the potassium solution which was placed coincided with a flat and concave reflective mirror, starting with zero shift. The fiber optic bundle was placed on an altered micrometer position shifted every fifty $\mu \mathrm{m}$. A certain shift position will get the maximum output voltage detected by the measured optical detector and obtain the reflected wave intensity data through the optical fiber receiver with the output voltage conversion of the detector as a function of fiber bundle shift. This measurement was conducted on all samples of six variations in concentration, including $0,2,4,6,8$, and $10 \mathrm{ppm}$. The next stage was continued for a solution of banana milk and green banana extracts.

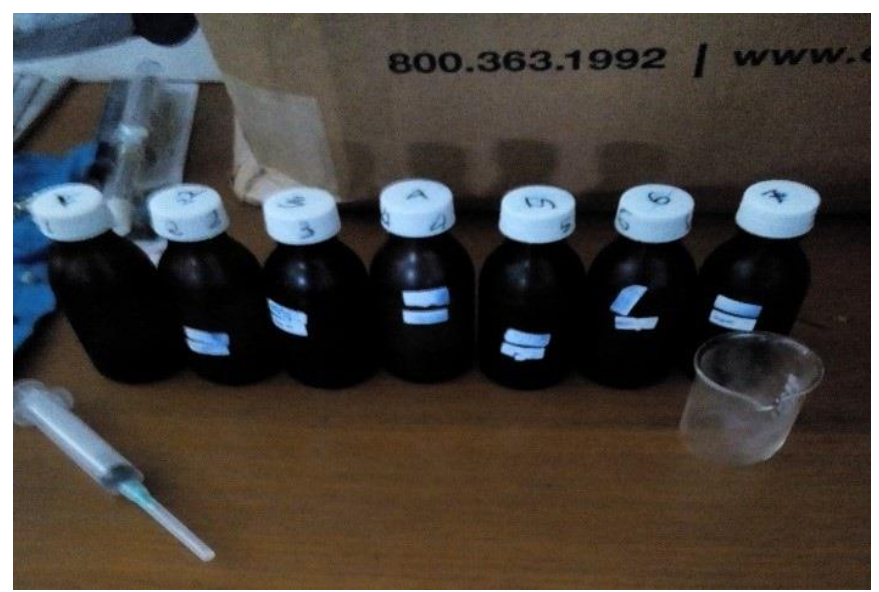

Figure 2. Sample 0, 2, 4, 6, 8, and 10 ppm standard potassium solution, milk banana extract solution and green banana extract

\section{Result and Discussion}

The parameters of wave intensity and output power to determine the potassium content of the standard potassium content sample indicate that the output power of the receiving fiber on the optical detector increases with an increase in the shift of the potassium sample from the optical fiber. This change is due to the increasing trend of the voltage until it reaches the maximum output voltage. When the change reaches its maximum peak, the output intensity decreases exponentially with the change in the shift of the optical fiber towards the potassium solution. This process shows that the reading of a digital voltmeter with the maximum output voltage of the change in six samples has decreased with increasing levels of potassium due to changes in the absorption of laser wave energy by the solution. The increasing of the concentration of the solution from the sample used, the greater energy absorption which causes the maximum voltage to decrease as shown in Table 1 . The concentration of the potassium solution varies from 0 , $2,4,6,8$, and $10 \mathrm{ppm}$ the output voltage starts at a minimum distance. The increase in distance to the sample solution affects the increase in the value of the output voltage until it reaches the maximum output voltage at the optical fiber shift distance of $850 \mu \mathrm{m}$. 
Table 1. The Maximum Output Voltage of the Variation in the Potassium Concentration

\begin{tabular}{llll}
\hline Data & $\begin{array}{l}\text { Potassium } \\
\text { Concentration } \\
(\mathbf{p p m})\end{array}$ & $\begin{array}{l}\text { Output } \\
\text { Voltage on a } \\
\text { Flat Mirror } \\
(\mathbf{m V})\end{array}$ & $\begin{array}{l}\text { Output Voltage } \\
\text { on a Concave } \\
\text { Mirror } \\
(\mathbf{m V})\end{array}$ \\
\hline 1 & 0 & 349.4 & 318.7 \\
2 & 2 & 349.1 & 317.4 \\
3 & 4 & 349.0 & 315.6 \\
4 & 6 & 348.8 & 315.4 \\
5 & 8 & 348.4 & 315.1 \\
6 & 10 & 348.2 & 315.0 \\
\hline
\end{tabular}

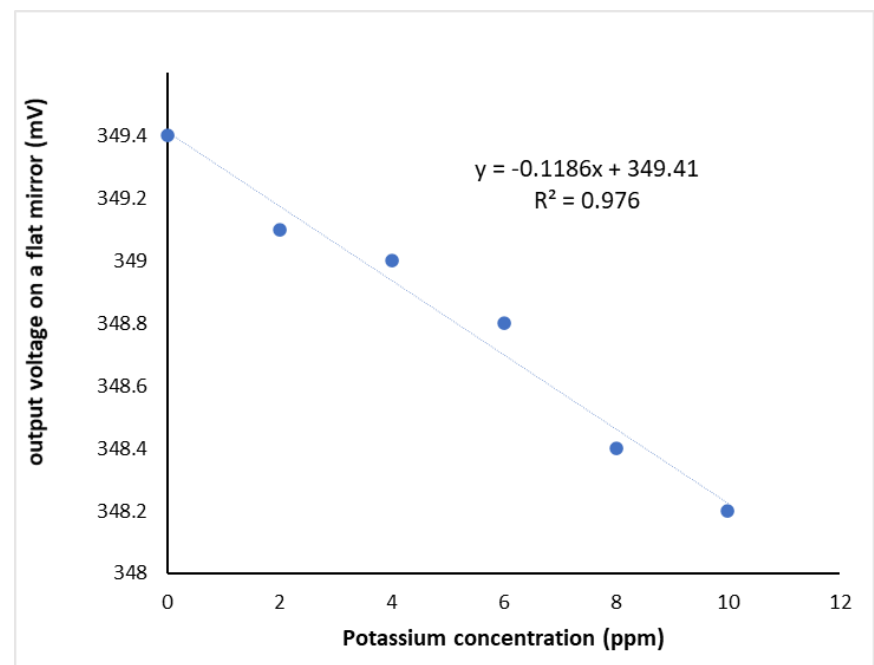

Figure 3. The maximum output voltage of the plane of the reflection of the flat mirror to the variation in the concentration of potassium

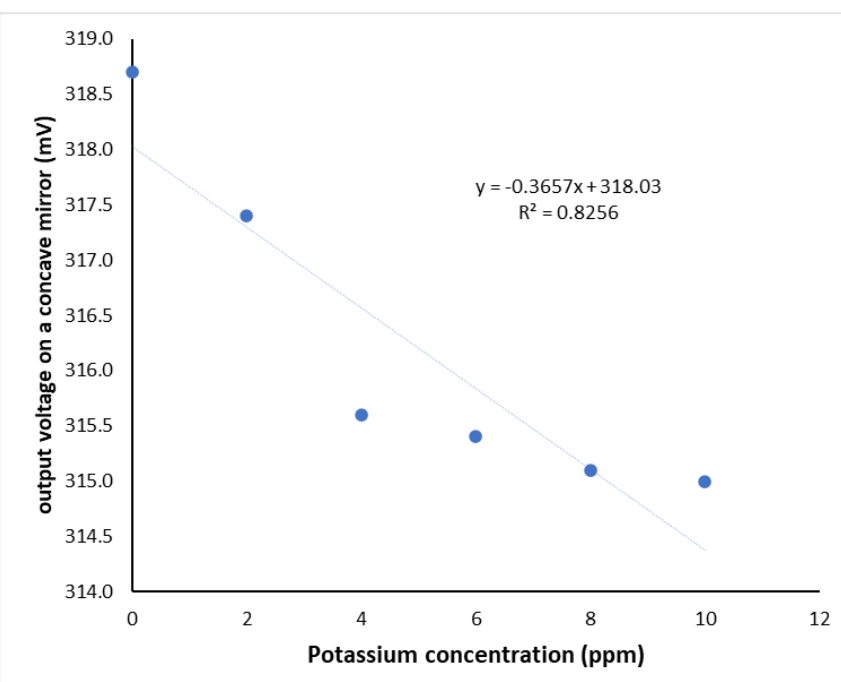

Figure 4. The maximum output voltage of the reflection plane of the concave mirror with respect to variations in the concentration of potassium

Figure 3 shows that the maximum output voltage shows a significant and linear decrease in the variation of the concentration of potassium solution which increases with high sensitivity and linearity. These results obtained on measurements with an optical sensor using a plane mirror plane reflection. Figure 4 shows the results of the measurement data for potassium concentration with the same process and parameter acquisition using a concave mirror reflection plane on the optical sensor. If we compare the results obtained from the two types of mirrors based on the measured output voltage (Figure 5), give a slight difference result. The plane mirror is more precise $(97.60 \%)$ than the concave mirror $(82.56 \%)$.

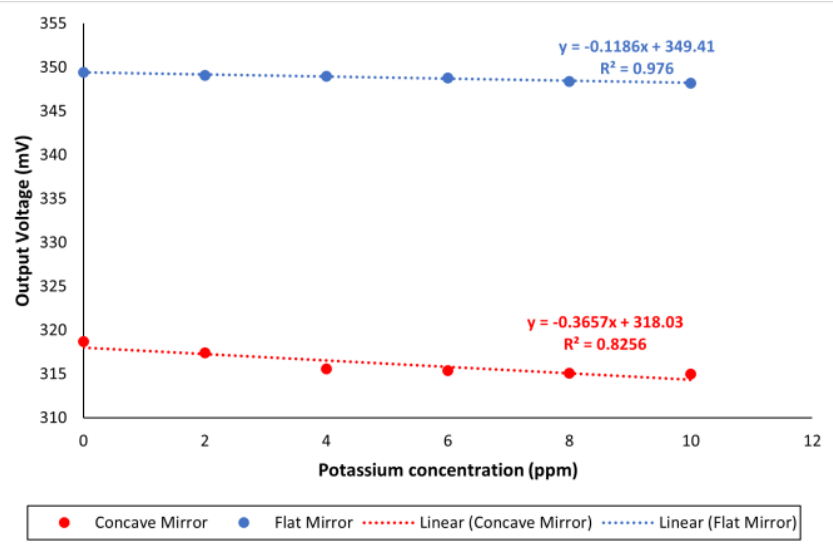

Figure 5. Comparation output voltage results based on potassium concentration toward reflective surface mirror types (plane and concave mirror)

Based on the results of the analysis of the maximum output voltage on a digital voltmeter against six variations of potassium concentrations using an optical sensor with a flat mirror reflection plane showing a sensitivity of $0.12 \mathrm{mV} / \mathrm{ppm}$ and a linearity of $97.6 \%$. This sensitivity analysis data shows that there is an approach to the sensitivity value using a quasiGaussian file. The results of data analysis determine the concentration of potassium using fiber bundles in accordance with the analysis obtained theoretically (Rahman et al., 2012). Measurement of the concentration of potassium using an optical sensor with a concave mirror reflection plane obtained a sensitivity of $0.36 \mathrm{mV} / \mathrm{ppm}$ and a linearity of $82.56 \%$. The results of the analysis of the optical sensors of the two reflective planes, both flat and concave mirrors, have the highest and most accurate linearity values found in the results of the plane of the flat mirror. The next step will be to measure the maximum output voltage read on an optical detector through an optical sensor with a sample of extracts of milk banana and green banana. The linear equation that becomes the reference is taken the results of the equation on the optical sensor in the plane of the reflection of the flat mirror with the consideration that the linearity value is higher and accurate with the equation $y=-0.1186 x+349.41$. The maximum output voltage measurement results with an 
optical detector on the optical sensor system obtained a voltage value of $349.9 \mathrm{mV}$ for green bananas, while for milk bananas it was obtained $355.2 \mathrm{mV}$. By analyzing the maximum output voltage value in the linear equation, the potassium content in milk bananas is $391.54 \mathrm{ppm}$ and the potassium content in the green banana extract solution is obtained $307.91 \mathrm{ppm}$.

\section{Conclusion}

Measurement of potassium levels in banana extract solution using an optical sensor was conducted using a standard potassium sample reflected by a flat mirror and a concave mirror. The use of these two different reflected plane mirrors to produce a high linearity value in one of the mirrors. The results of measurement analysis using an optical sensor with a concave mirror reflection plane obtained a sensitivity of $0.36 \mathrm{mV} / \mathrm{ppm}$ and a linearity of $82.56 \%$. In the plane of the flat mirror reflection, obtained an optical sensor with a plane mirror reflection plane shows a sensitivity of $0.12 \mathrm{mV} / \mathrm{ppm}$ and a linearity of $97.6 \%$. The analysis of the optical sensors of the two reflection planes, both flat mirrors and concave mirrors, has the highest and most accurate linearity values found in the plane mirror reflection plane results. Then proceed to the stage of measuring the maximum output voltage read on the optical detector through an optical sensor with a sample of extracts of milk bananas and green bananas. The results of data analysis on the linear equation with the highest linearity obtained the potassium content in milk bananas of $391.54 \mathrm{ppm}$ and the potassium content in the green banana extract solution was obtained $307.91 \mathrm{ppm}$. The potassium content in the milk banana extract is higher than the green banana with a linearity of more than $97 \%$.

\section{References}

do Prado Ferreira, M., \& Teixeira Tarley, C. R. (2020). Assessment of in vitro bioacessibility of macrominerals and trace elements in green banana flour. Journal of Food Composition and Analysis, $\quad 92,103586$. https://doi.org/10.1016/i.jfca.2020.103586

Hida, N., Bidin, N., Abdullah, M., \& Yasin, M. (2013). Fiber optic displacement sensor for honey purity detection in distilled water. Optoelectronics and Advanced Materials-Rapid Communications, 7(7-8), 565-568. Retrieved from https://oamrc.inoe.ro/articles/fiber-optic-displacementsensor-for-honey-purity-detection-in-distilledwater/

Horie, K., Hossain, M. S., Morita, S., Kim, Y., Yamatsu,
A., Watanabe, Y., ... Kim, M. (2020). The potency of a novel fermented unripe banana powder as a functional immunostimulatory food ingredient. Journal of Functional Foods, 70, 103980. https://doi.org/10.1016/j.jff.2020.103980

Qiu, J., Zhang, Y., Dong, C., Huang, Y., Sun, L., Ruan, H., .. Wu, A. (2019). Rapid colorimetric detection of potassium ions based on crown ether modified $\mathrm{Au}$ NPs sensor. Sensors and Actuators B: Chemical, 281, 783-788. https://doi.org/10.1016/j.snb.2018.10.139

Rahman, H. A., Harun, S. W., Yasin, M., \& Ahmad, H. (2012). Fiber-optic salinity sensor using fiberoptic displacement measurement with flat and concave mirror. IEEE Journal of Selected Topics in Quantum Electronics, 18(5), 1529-1533. https://doi.org/10.1109/JSTQE.2011.2159705

Rahman, H. A., Harun, S. W., Yasin, M., \& Ahmad, H. (2013). Fiber optic salinity sensor using beamthrough technique. Optik - International Journal for Light and Electron Optics, 124(8), 679-681. https://doi.org/10.1016/j.ijleo.2012.01.020

Samian, Zaidan, A. H., Sujito, Yasin, M., Pujiyati, M., \& Supadi. (2018). Liquid level sensor using two fiber bundles. Sensors and Actuators A: Physical, 280 , 552-558. https://doi.org/10.1016/j.sna.2018.08.032

Su, H., Ruan, W., Ye, S., Liu, Y., Sui, H., Li, Z., ... Zhao, B. (2016). Detection of physiological potassium ions level in human serum by Raman scattering spectroscopy. Talanta, 161, 743-747. https://doi.org/10.1016/j.talanta.2016.09.010

Susilawati, Doyan, A., Taufik, M., \& Wahyudi. (2018). Synthesis and characterization of Barium MHexaferrite with metal doping $\mathrm{Mn}$ and $\mathrm{Ni}$ for microwaves absorbent. Journal of Physics: Conference Series, 1120, 012002. https://doi.org/10.1088/17426596/1120/1/012002

Yasin, M., Soelistiono, S., Yhun Yhuwana, Y. G., Khasanah, M., Arof, H., Irawati, N., \& Harun, S. W. (2015). Intensity based optical fiber sensors for calcium detection. Optoelectronics and Advanced Materials-Rapid Communications, 9(910), 1185-1189. Retrieved from https://oamrc.inoe.ro/articles/intensity-based-optical-fibersensors-for-calcium-detection/ 